\title{
PROTECTION OF CHINESE HERBS AGAINST ADENINE-INDUCED CHRONIC RENAL FAILURE IN RATS
}

*Yanqing Tong, M.D., ${ }^{2}$ Bing Han, M.D., ${ }^{3}$ Hongyang Guo, M.D. and ${ }^{4}$ Yanru Liu, M.D.

Department of Nephrology, The First Affilliated Hospital to Changchun University of Chinese Medicine, Changchun city, Jilin province, China., ${ }^{2}$ Department of Nephrology, The First Affilliated Hospital to Changchun University of Chinese Medicine, Changchun city, Jilin province, China., ${ }^{3}$ Department of Nephrology, The First Affilliated Hospital to Changchun University of Chinese Medicine, Changchun city, Jilin province, China. ${ }^{4}$ Department of Nephrology, The First Affilliated Hospital to Changchun University of Chinese Medicine, Changchun city, Jilin province, China.

*Email: tyq1229@yahoo.com.cn

\begin{abstract}
The aim of the study is to evaluate the efficacy of Chinese herbs (Angelica sinensis, Ligusticum wallichii, Salvia miltiorrhiza, Rhizoma dioscoreae, Rhodiola crenilata, Astragalus membranaceus and Angelica sinensis) on adenine-induced chronic renal failure in rats. 30 age-matched male Wistar rats were divided into three groups. Rats in group A (n=10), B $(\mathrm{n}=10)$ and $\mathrm{C}(\mathrm{n}=10)$ were fed a standard laboratory chow and allowed tap water ad libitum. In group B and $\mathrm{C}$, renal failure was induced by the administration of a diet containing $0.75 \%$ adenine for 28 days which began at day 0 . Rats in group C were given Chinese herbs $(40 \mathrm{ml} / \mathrm{kg}$ with drug concentration $1.75 \mathrm{~g} / \mathrm{ml})$ beginning at day 0 . Urine albumin, blood urea nitrogen (BUN) and creatinine were determined at days 0, 14 and 28. At day 28, the animals were killed and their kidneys removed for light microscope evaluation. Body weight in Group B decreased more significantly than that in Group C $(\mathrm{p}=0.032)$ at day 28 . The rats in group B demonstrated more severe proteinuria and higher Serum creatinine and BUN levels than group $\mathrm{C}$ at day 14 and day $28(\mathrm{P}<0.05,0.01)$. All rats given adenine developed marked structural renal damage involving the tubule and interstitium. The values were much less severe in group $\mathrm{C}$ than those in group B. In adenine-induced chronic renal failure rats, the protective effects of these Chinese herbs were of a significant nature. Our results do support the notion that these Chinese herbs are useful in deferring the advance of chronic renal failure. We recommend Chinese herbs as a beneficial treatment for pre-end stage chronic renal failure.
\end{abstract}

Key words Chinese herbs; Adenine; Renal failure; Histology.

\section{Introduction}

Many renal diseases in humans are progressive in nature and eventually result in renal failure. As a result, the number of patients with renal failure continues to increase worldwide. Therefore, there is a need for the development of new effective therapeutic strategies. For the generation of new therapeutic strategies, it is essential to use animal models that simulate clinical manifestations of renal diseases in humans. To be clinically useful, we believe that such a model should simulate human renal diseases in their natural course as well as in histological features, predictors of renal functional outcome and responsiveness to drugs used clinically.

Progressive chronic renal failure is characterized histologically by tubulointerstitial and vascular scarring. Recently, adenine-induced renal failure rats were used as the disease-model for evaluation of drug efficacy (Lacour et al., 2005). Orally administered adenine is immediately metabolized to 2,8-dihydroxyadenine, which formed crystals in the apical region of the proximal tubular epithelia. Increased crystals induced damages in the cells of these tissues and caused renal dysfunction with 
increased levels of blood urea nitrogen (BUN) and creatinine (Koeda et al., 1988; Yokozawa et al., 1986). If the 0.75\% adenine feeding period was continued for four weeks or more, irreversible renal failure was induced and the deteriorating renal biochemical parameters did not recover (Katsumata et al., 2003). Therefore, the rats orally administrated $0.75 \%$ adenine for more than four weeks are considered to be a model of rapidly progressive type of chronic renal failure, which are more compatible with the clinical findings (Keiichi et al., 2006).

Traditional Chinese medicine (TCM) has evolved into a well-developed, coherent system of medicine that uses several modalities to treat and prevent illness. The most commonly employed therapeutic methods in TCM include Chinese herbal medicine, acupuncture/moxibustion, diet therapy, mind/body exercises (Qigong and Tai Chi), and Tui Na (Chinese massage) (Lao, 1999). Herbal medicine has been an integral part of TCM for more than 2000 years. Many herbal formulations have been developed and are used in the treatment of pre-end stage renal failure. In our preliminary study, favorable results were obtained in several pre-end stage renal failure patients administered some Chinese herbs, in whom a significant reduction of BUN and creatinine was seen (Tong, 2007). In an attempt to evaluate these Chinese herbs' efficacy, we have studied their action in the animal model of adenine-induced renal failure.

\section{Methods and Materials}

\section{Animals}

The study was approved by the local ethical committee and referred to the National Research Council Guide for the Care and Use of Laboratory Animals. Experiments were performed using 30 age-matched male Wistar rats (purchased from Jilin University, Changchun, China) of mean body weight $232 \mathrm{~g}$ (range 180-280g). The rats were maintained in individual cages at a temperature-controlled room $\left(22^{\circ} \mathrm{C}\right)$ on a 12:12-h light-dark cycle (light at 07:00). They were fed a standard laboratory chow $(0.35 \mathrm{~g} \mathrm{NaCI}, 20 \mathrm{~g}$ protein) and allowed tap water ad libitum (group A, B and C). Body weight (BW) and food intake were recorded daily.

\section{Induction of Renal Failure}

In group $\mathrm{B}(\mathrm{n}=10)$ and $\mathrm{C}(\mathrm{n}=10)$, renal failure was induced by the administration of a diet containing $0.75 \%$ adenine (Sigma, St Louis, MO, USA) for 28 days began at day 0 .

\section{Drug therapy}

The rats in group A and B were given only water ad libitum. Rats in group $\mathrm{C}$ were given Chinese herbs at an average dose of $40 \mathrm{ml} / \mathrm{kg}$ beginning at day 0 .

The Chinese herbs administered were as follow: Da Huang [Angelica sinensis (Oliv.) Diels] 15g, Chuan Xiong [Ligusticum wallichii Franch.] 15g, Dan Shen [Salvia miltiorrhiza Bge.] 15g, Bi Xie [Rhizoma Dioscoreae Septemlobae] 15g, Hong Jingtian [Rhodiola crenilata (Hook. F. et Thoms.)H. Ohba] 15g, Huang Qi [Astragalus membranaceus (Fisch.) Bge. var. mongho.] 40g, Dang Gui [Angelica sinensis (Oliv.) Diels] 15g. The seven crude drugs were mixed in 800ml water, producing $100 \mathrm{ml}$ liquor after decoction in $800 \mathrm{ml}$ water $\left(100^{\circ} \mathrm{C}\right.$ for 30 mins twice). The liquor after filtration gave $1.75 \mathrm{~g} / \mathrm{ml}$ as the drug concentration.

\section{Urinary albumin excretion}

All the rats were weighed and placed in metabolic cages at days 0,14 and 28, to determine albumin excretion in 24-hr urine. Urine albumin was determined with a nephelometric method (Boehring Nephelometer Analyzer, Behringwerken AG, 
Marburg, Germany).

\section{Serum BUN and creatinine analysis}

The blood was collected for the measurement of Serum creatinine and BUN. At days 0 and 14, the blood was collected via the tail vein, and by cardiac puncture at day 28. Serum creatinine and BUN were measured using an autoanalyzer (TBA-80FR, Toshiba, Tokyo, Japan).

\section{Kidney histologic analysis}

At day 28, the animals were killed by cervical dislocation, followed by immediate organ collection for histologic analysis of kidneys. Their kidneys removed, preserved in formalin and subsequently embedded in paraffin. Large sections (1 $\mathrm{mm}$ ) were cut perpendicularly to the renal capsule, in order to ensure that both cortex and medulla would be present in each section. Samples were stained with haematoxylin-eosin dye and selected randomly for light microscope evaluation.

\section{Statistical analyses}

Mean values \pm standard error of mean (SEM) or range with median values are given. The paired t-test was used.

\section{Results}

Animal characteristics

The administration of adenine decreased food intake, especially during the last two weeks because rats in group B ate only $15-25 \%$ of the pre-administration intake (20-25g). However, food intake of Group C animals was $47-53 \%$ of those observed before the adenine administration. In this study, BW was similar in the three groups at the beginning of the study. After the 4-wk experimental period, BW in Group B decreased more significantly than that in Group C (-28 \pm 3 and $-15 \pm 2$, respectively, $\mathrm{p}=0.032$, paired $\mathrm{T}$ test $)$, with almost no change in group $\mathrm{A}(3 \pm 2)$.

\section{Urinary albumin excretion}

The rats in groups A show no proteinuria during the whole experimental process. Rats in groups B demonstrated severe susceptibility to adenine administration with the onset of proteinuria at day 14 and day 28 . The rats in group $\mathrm{C}$ were partly resistant to adenine with decreasing proteinuria after therapy (Table 1).

\section{BUN and creatinine changes}

Serum creatinine and BUN levels were higher in group B and C compared to the respective control group A $(p<0.01)$. At day 14 and 28, the highest values were in group B (Table 1). We do not have creatinine clearances, but since serum creatinine was the same in the different groups at day 0 , the degree of real insufficiency must have been similar. 
Table 1: Urinary albumin, BUN and creatinine levels of all groups ${ }^{\mathrm{a}}$

\begin{tabular}{|c|c|c|c|}
\hline groups & $\begin{array}{l}\text { Urinary albumin } \\
\qquad(\mathrm{mg} / 24 \mathrm{~h})\end{array}$ & $\begin{array}{c}\text { Serum BUN } \\
(\mathrm{mg} / \mathrm{dl})\end{array}$ & $\begin{array}{l}\text { Serum creatinine } \\
(\mathrm{mg} / \mathrm{dl})\end{array}$ \\
\hline \multicolumn{4}{|l|}{ group A } \\
\hline \multirow[t]{2}{*}{ day 0} & $0 \pm 0$ & $8.06 \pm 2.38$ & $0.55 \pm 0.11$ \\
\hline & (10) & (10) & (10) \\
\hline \multirow[t]{2}{*}{ day 14} & $0 \pm 0$ & $8.22 \pm 4.75$ & $0.52 \pm 0.10$ \\
\hline & (10) & (10) & (10) \\
\hline \multirow[t]{2}{*}{ day 28} & $0 \pm 0$ & $8.10 \pm 3.20$ & $0.53 \pm 0.15$ \\
\hline & (10) & (10) & (10) \\
\hline \multicolumn{4}{|l|}{ group B } \\
\hline \multirow[t]{2}{*}{ day 0} & $0 \pm 0$ & $8.20 \pm 3.11$ & $0.52 \pm 0.12$ \\
\hline & (10) & (10) & (10) \\
\hline \multirow[t]{2}{*}{ day 14} & $165.2 \pm 22.8^{\mathrm{b}}$ & $86.25 \pm 10.15^{\mathrm{b}}$ & $0.87 \pm 0.25^{\mathrm{c}}$ \\
\hline & (10) & (10) & (10) \\
\hline \multirow[t]{2}{*}{ day 28} & $236.5 \pm 28.6^{\mathrm{b}}$ & $117.50 \pm 26.46^{\mathrm{b}}$ & $0.98 \pm 0.30^{\mathrm{b}}$ \\
\hline & (9) & (9) & (9) \\
\hline \multicolumn{4}{|l|}{ group C } \\
\hline \multirow[t]{2}{*}{ day 0} & $0 \pm 0$ & $8.15 \pm 3.68$ & $0.53 \pm 0.08$ \\
\hline & (10) & (10) & $(10)$ \\
\hline \multirow[t]{2}{*}{ day 14} & $82.2 \pm 15.0^{\mathrm{b}, \mathrm{d}}$ & $55.30 \pm 12.55^{\mathrm{b}, \mathrm{d}}$ & $0.66 \pm 0.17^{\mathrm{e}}$ \\
\hline & (10) & (10) & $(10)$ \\
\hline \multirow[t]{2}{*}{ day 28} & $115.0 \pm 19.5^{\mathrm{b}, \mathrm{d}}$ & $70.16 \pm 20.05^{\mathrm{b}, \mathrm{e}}$ & $0.70 \pm 0.13^{\mathrm{c}, \mathrm{e}}$ \\
\hline & (10) & (10) & (10) \\
\hline
\end{tabular}

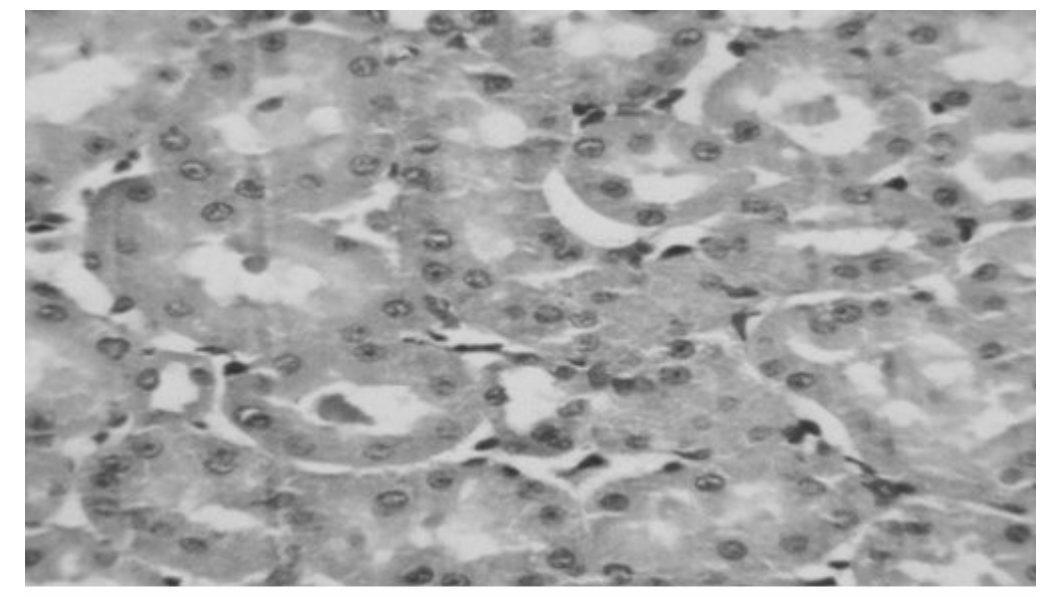

Fig.1. Normal tubular cells and tubules in group A at day 28 $\mathrm{HE}, 80 \times$.

\section{Renal pathology}

Twenty-eight rats were available for renal pathology study. Normal renal pathology in group A animals is shown in 
Figure 1. All rats given adenine developed marked structural damage, usually involving the tubule and interstitium. The damages included tubular dilatation, epithelial hyperplasia, calcification of the basement membrane of tubules, crystals of adenine in the interstitium, inflammatory cellular infiltration and giant cells in interstitium. All the values were much less severe in group C than those in group B (Figures 2-4).

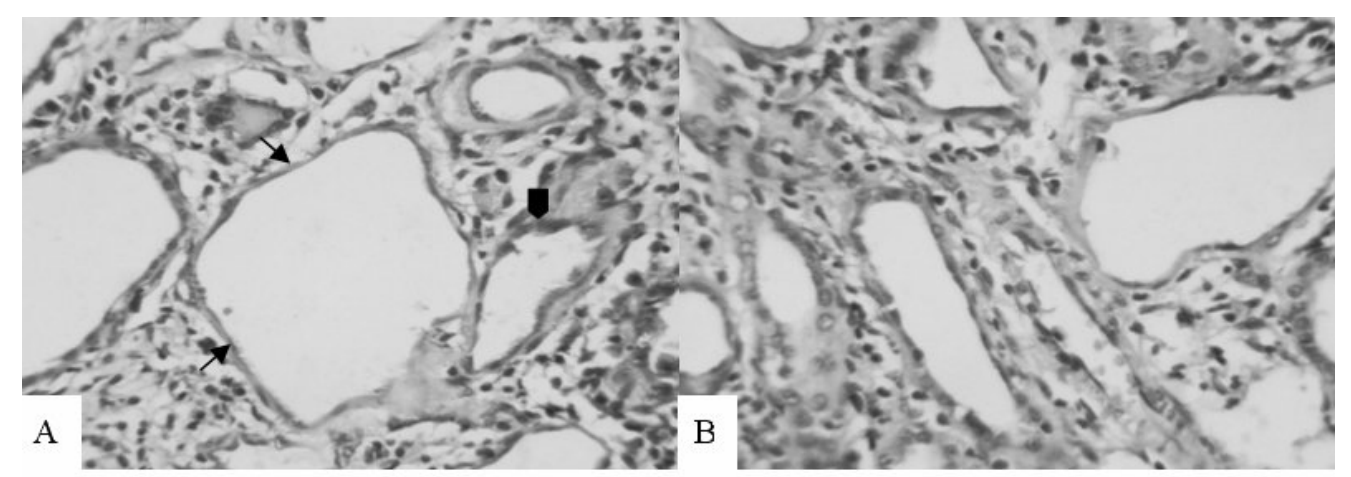

Fig.2. Tubular damages. (A) Tubular dilatation (arrows), epithelial hyperplasia, calcification of the basement membrane of tubules (arrowhead) in group B at day 28, HE, 80×. (B) The rats in group $\mathrm{C}$ show less severe tubular damage at day $28, \mathrm{HE}, 80 \times$.

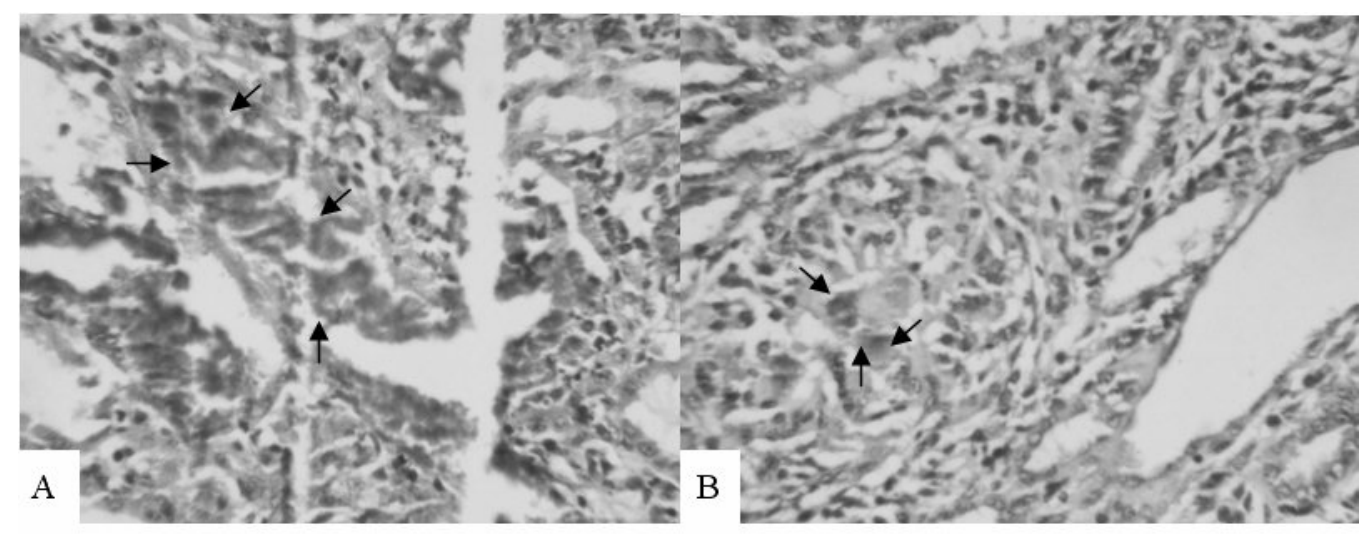

Fig.3. Crystals of adenine in the interstitium. (A) Massive crystals of adenine in the interstitium (arrows) in group B at day $28, \mathrm{HE}, 80 \times$. (B) Much less crystals of adenine in the interstitium (arrows) in group $\mathrm{C}$ at day $28, \mathrm{HE}, 80 \times$.

\section{Discussion}

All the Chinese herbs in the experiment were shown to be valuable for pre-end stage of renal disease previously. These properties have prompted their compound prescription use in the management of humans and experimental animals with renal failure. With that in mind, we used adenine-induced chronic renal failure rats in order to analyze kidney histology and to investigate the effects of Chinese herbs on adenine-induced renal injury. 


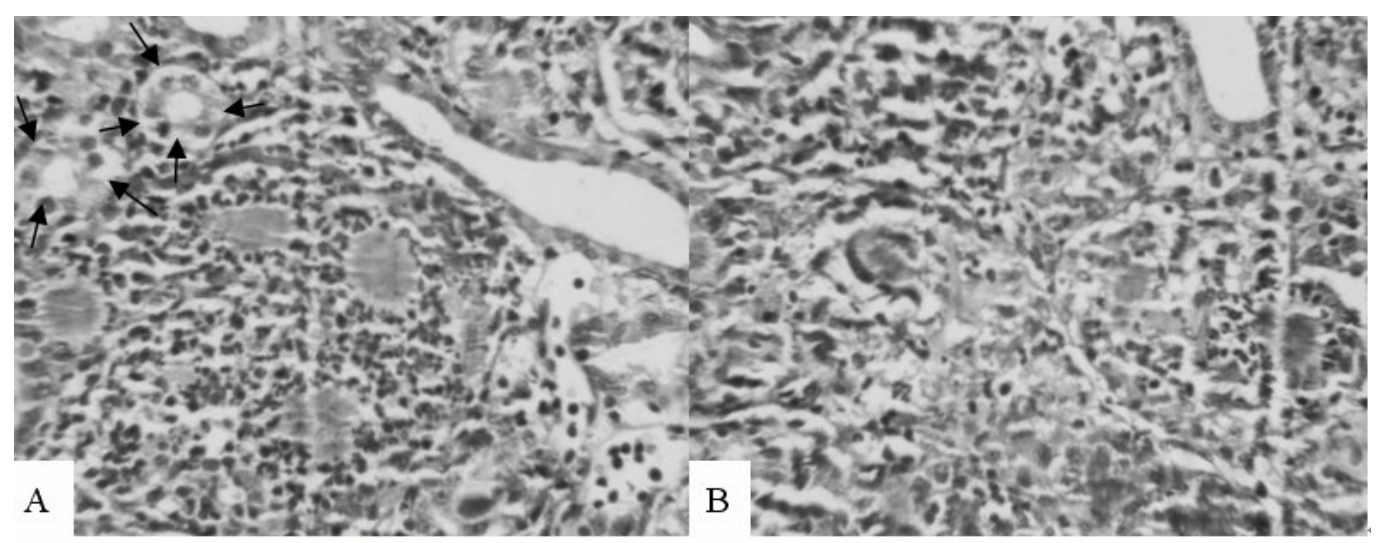

Fig.4. Inflammatory cell infiltration. (A) Severe inflammatory cell infiltration and giant cells (arrows) in interstitium of the kidney in group B at day 28, HE, 80×. (B) Less inflammatory cell infiltration in the interstitium in group $\mathrm{C}$ at day $28, \mathrm{HE}, 80 \times$.

In our study, the adenine-induced renal failure in rats, the animal model we used, resembled chronic renal failure in humans, both biochemically and morphologically. The characteristic histological features of our models were findings of a scattered distribution of damaged tubules. Development of massive albuminuria and an increase in the serum concentration of creatinine and BUN, resemble the clinical course of progressive human kidney diseases. We believe that this model is particularly suitable for testing new therapy, because of the rapid course and the fact that there is little variation between individual rats. Thus any beneficial effects of treatment should be detected using a minimum number of animals.

To further explore the effect of Chinese herbs, we carried out the study in an attempt to validate the protective action of the drug. In the study, the administration of adenine was associated with malnutrition, essentially due to a reduction of food intake. We are able to show a significant recovery of food intake and progressive decrease of proteinuria, serum creatinine and BUN after Chinese herbs administration in adenine-fed rats. During the 4 weeks of its administration, we have also observed that these Chinese herbs attenuated the histologic severity of kidney.

Even if a treatment is efficacious, it may not be possible to determine the mechanism of action and the active substances immediately, particularly in herbal treatments that contain many compounds. Many powerful interventions, such as penicillin and smallpox vaccination, were accepted well before their mechanisms were understood (Wynder, 1994). So lack of knowledge about mechanisms and active substances need not prevent the use of efficacious treatments. In this case, the multi-herb formula was prepared in decocted extracts. One has to expect the possibility that some chemical interactions take place among natural constituents existing in the component herbs of the formula during decoction. Decoction may change the extraction rates of the active ingredients and/or produce new artificial substances, which may then exhibit new pharmacological activities. However, a mixture of several crude extracts could have greater beneficial effects compared with a single plant extract if properly used. First, crude herbs given in combination could act synergistically. Second, they could have unknown interactions but could interact to diminish possible adverse side effects of one or more of the components. Finally, their combination could prevent the gradual decline in effectiveness observed when single herbs are given over long periods of time (Matsuo et al. 1994).

So we can only speculate on the mechanism underlying the protective effect of these Chinese herbs on experimental renal failure. The components of the drug could prove beneficial through a wide range of actions. For instance, Huang Qi and Dang Gui have antioxidant (Sun et al., 1996) and antifibrosis effects (Meng et al., 2006). Chuan Xiong has antiproliferative effect (Xiao et al. 2004). Da huang and Dan Shen can improve the renal function and prevent the development of renal failure, which may be related to the inhibition of endogenous endothelin release (Xu et al. 2001) and the inhibition of the expressions of Platelet-derived growth factor (PDGF) and basic fibroblast growth factor (bFGF) (Yang et al. 2007).

In China, traditional medicinal herbs are often used for clinical and experimental chronic renal disease (YP Chen, 
1986; Wang et al. 1996). Chinese herbs are also used in conjunction with Western pharmacological agents (Liu et al., 1991; Fu et al., 2004; Zhang et al., 1994). Such an approach has also been advocated for the management of experimental and clinical chronic renal failure. In our animal model, the protective effects of these Chinese herbs were of a significant nature. Our results in this particular experimental model, do support the notion that these Chinese herbs are useful in deferring the advance of chronic renal failure. However, there is still a great need for more and better research on the efficacy and safety of these Chinese herbs.

\section{Conclusions}

Although it is difficult to extrapolate animal data to humans, we recommend these Chinese herbs as a beneficial treatment for pre-end stage chronic renal failure.

\section{Acknowledgments}

The research was funded by the Administration of Traditional Chinese Medicine. We thank Zei He M.D., Pingfu Liu M.D. for helpful assistance in preparing the manuscript.

\section{References}

1.Chen YP. (1986). Treatment of chronic renal failure with traditional Chinese medicine. Follow-up of 90 cases. Zhong Xi Yi Jie He Za Zhi. 6: 465-467, 452.

2.Chen YP. (1992). Symposium on the treatment of renal failure with traditional Chinese medicine combined with Western medicine. Zhongguo Zhong Xi Yi Jie He Za Zhi. 12: 576-inside backcover.

3.Fu P, Yu G, Huang X, Xu J, Mei X, Wu Hao. (2004). Effect of integrated traditional Chinese and Western medicine therapy on progression of acute renal failure in patients with chronic renal insufficiency: a short-term clinical study. Zhong Xi Yi Jie He Xue Bao. 2: 429-431.

4.Katsumata K, Kusano K, Hirata M, Tsunemi K, Nagano N, Burke SK. (2003). Sevelamer hydrochloride prevents ectopic calcification and renal osteodystrophy in chronic renal failure rats. Kidney Int. 64: 441-450.

5.Keiichi T, Qunsheng Y, Hiroyuki O, Ikuo I, Yoshiyuki M, Nobuo I. (2006). Severe hyperparathyroidism with bone abnormalities and metastatic calcification in rats with adenine-induced uraemia. Nephrol Dial Transplant. 21: 651-659.

6.Koeda T, Wakaki K, Koizumi F, Yokozawa T, Oura H. (1988). Early changes of proximal tubules in the kidney of adenine-ingesting rats, with special reference to biochemical and electron microscopic studies. Nippon Jinzo Gakkai Shi. 30: 239-246.

7.Lacour B, Lucas A, Auchere D, Ruellan N, de Serre Patey NM, Drüeke TB. (2005). Chronic renal failure is associated with increased tissue deposition of lanthanum after 28-day oral administration. Kidney Int. 67: 1062-1069.

8.Lao L. (1999). Traditional Chinese Medicine. In Essentials of Complementary and Alternative Medicine. Jonas WB, Levin JS, Eds. Baltimore, Md., Lippincott Williams and Wilkins: 216-232.

9.Liu JB, Liu JX, Jin SL. (1991). Traditional Chinese medicine combined with Western medicine in the treatment of severe renal failure in patients with epidemic hemorrhagic fever. Zhong Xi Yi Jie He Za Zhi. 11: 475-476, 453.

10. Matsuo R, Ball MA, Kobayashi M, Herndon DN, Pollard RB, Suzuki F. (1994). Effects of a traditional Chinese herbal medicine, Kanzo-Bushi-To, on the resistance of thermally injured mice infected with herpes simplex virus type 1. Int J Immunopharmacol. 16: 855-863.

11. Meng LQ, Qu L, Li X. (2006). Astragalus and Angelica mixture ameliorates renal interstitial fibrosis by a multiple 
manner. Chinese Pharma Bulletin. 22: 296-302.

12. Sun C, Jiang Y, Zhong G, Huanxin L. (1996). Study on antioxidant effect of Astragalus polysaccharide. Chinese Pharma Bulletin. 12: 161-163.

13. Tong Y. (2007). Clinical Observation on the effect of the Method of Clearing the Upper for Treating the Lower in Treatment of Chronic Glomerulonephritis. J Tradit Chin Med. 48: 699-701.

14. Wang L, Wang MZ. (1996). Survey on experimental studies on traditional Chinese medicine treatment of chronic renal failure. Zhongguo Zhong Xi Yi Jie He Za Zhi. 16: 58-60.

15. Wynder EL. (1994). Studies in mechanism and prevention: striking a proper balance. Am J Epidemiol. 139: 547-549.

16. Xiao J, Yi Z, Wu X. (2004). Effect of Tetramethylpyrazine Injection on Proliferation of Glomerulus Mesangium Cells in Rats. Herald Med. 23: 611-613.

17. Xu M, Wang Y, Sun W, Xuan L, Xu Y. (2001). Effect of salvianolate on renal function and endogenous endothelin release in chronic renal failure rats. Chinese J.Pharmacol. Toxicol. 15: 39-42.

18. Yang Y, Han S, Han Y, Yuan Z, Yang M. (2007). Effects of Da Huang Huo Ling Tang on the expression of PDGF, bFGF in Rats with Renal failure caused by Adenine. J Sichuan Tradit Chin Med. 25: 26-28.

19. Yokozawa T, Zheng PD, Oura H, Koizumi F. (1986). Animal model of adenine-induced chronic renal failure in rats. Nephron. 44: 230-234.

20. Zhang SZ, Zhu CF, Wang YJ. (1994). Observation on 104 senile chronic renal insufficiency patients treated with integrated traditional Chinese and Western medicine. Zhongguo Zhong Xi Yi Jie He Za Zhi. 14: 279-281, 260. 